\title{
A Vaccinia Virus Encoding the Human Sodium Iodide Symporter Facilitates Long-Term Image Monitoring of Virotherapy and Targeted Radiotherapy of Pancreatic Cancer
}

\author{
Dana Haddad ${ }^{1,2}$, Pat B. Zanzonico ${ }^{3}$, Sean Carlin ${ }^{4}$, Chun-Hao Chen ${ }^{1}$, Nanhai G. Chen ${ }^{5,6}$, Qian Zhang ${ }^{5,6}$, Yong A. Yu ${ }^{5,6}$, \\ Valerie Longo ${ }^{3}$, Kelly Mojica ${ }^{1}$, Richard J. Aguilar ${ }^{5}$, Aladar A. Szalay ${ }^{2,5,6}$, and Yuman Fong ${ }^{1}$ \\ ${ }^{I}$ Department of Surgery, Memorial Sloan-Kettering Cancer Center, New York, New York; ${ }^{2}$ Department of Biochemistry, University of \\ Würzburg, Würzburg, Bavaria, Germany; ${ }^{3}$ Departments of Medical Physics and Radiology, Memorial Sloan-Kettering Cancer Center, \\ New York, New York; ${ }^{4}$ Radiopharmaceutical Chemistry Service, Department of Radiology, Memorial Sloan-Kettering Cancer Center, \\ New York, New York; ${ }^{5}$ Genelux Corp., San Diego Science Center, San Diego, California; and ${ }^{6}$ Department of Radiation Oncology, \\ Rebecca and John Moores Comprehensive Cancer Center, University of California, San Diego, California
}

To assess therapeutic response and potential toxicity of oncolytic virotherapy, a noninvasive, deep-tissue imaging modality is needed. This study aimed to assess the feasibility, parameters, and determining factors of serial imaging and long-term monitoring of virotherapy and the therapeutic response of pancreatic cancer xenografts treated with a vaccinia virus carrying the human sodium iodide symporter GLV-1h153. Methods: Pancreatic cancer xenografts (PANC-1) in nude mice were treated systemically or intratumorally with GLV-1h153 and serially imaged using ${ }^{124}$ I PET at 1, 2, 3, and 5 wk after viral injection. Signal intensity was compared with tumor therapeutic response and optical imaging, and tumors were histologically analyzed for morphology and the presence of virus. Autoradiography was performed using technetium-pertechnetate and $\gamma$-scintigraphy to assess determining factors for radiouptake in tumors. Finally, the enhanced therapeutic effect of combination therapy with GLV-1h153 and systemic radioiodine was assessed. Results: GLV-1h153 successfully facilitated serial long-term imaging of virotherapy, with PET signal intensity correlating to tumor response. GLV-1h153 colonization of tumors mediated radioiodine uptake at potentially therapeutic doses. Successful radiouptake required the presence of virus, adequate blood flow, and viable tissue, whereas loss of signal intensity was linked to tumor death and necrosis. Finally, combining systemically administered GLV$1 \mathrm{~h} 153$ and ${ }^{131} \mathrm{I}$ led to enhanced tumor kill when compared with virus or ${ }^{131}$ I alone $(P<0.01)$. Conclusion: GLV-1h153 is a promising oncolytic agent for the treatment, long-term imaging, and monitoring of therapeutic response in a xenograft model of pancreatic cancer. GLV-1h153 provided insight into tumor biologic activity and facilitated enhanced tumor kill when combined with systemic targeted radiotherapy. These results warrant further

Received Feb. 25, 2012; revision accepted Jul. 9, 2012

For correspondence or reprints contact either of the following:

Aladar A. Szalay, Genelux Corp., San Diego Science Center, 3030 Bunker

Hill St., Ste. 310, San Diego, CA 92109.

E-mail: aaszalay@genelux.com

Yuman Fong, Department of Surgery, Memorial Sloan-Kettering Cancer

Center, 1275 York Ave., New York, NY 10065.

E-mail: fongy@mskcc.org

Published online Nov. 8, 2012.

COPYRIGHT @ 2012 by the Society of Nuclear Medicine and Molecular Imaging, Inc. investigation into parameters and potential synergistic effects of combination therapy.

Key Words: oncolytic; vaccinia; pancreatic cancer; positron emission tomography; targeted radiotherapy

J Nucl Med 2012; 53:1933-1942

DOI: 10.2967/jnumed.112.105056

D espite advances in conventional therapy, the formidable challenge of treating cancer, especially if progressed, still remains. Pancreatic cancer in particular is the fourth leading cause of cancer death in the United States (1), and an objective response to single-agent or combination chemotherapies, which are never curative, occurs in less than $20 \%$ of patients $(1,2)$. These results emphasize the need for novel therapeutic strategies for this disease.

It is well established that combining several treatment modalities appears to provide the highest possibility of fighting this disease (3). Oncolytic viral therapies have emerged as a potential therapeutic option, with the possible advantage of lessening side effects and enhancing treatment efficacy because of high tumor selectivity $(3,4)$. Oncolytic vaccinia viruses (VACVs) in particular have shown promise in both preclinical and clinical trials (5). We have previously reported on the feasibility of using the genetically engineered vaccinia strain GLV-1h153 for the noninvasive imaging and treatment of pancreatic cancer (6). This virus has also been effective against other cancers, including anaplastic thyroid cancer (7). GLV-1h153 was genetically modified from GLV$1 \mathrm{~h} 68$ to express the human sodium iodide symporter (hNIS), an intrinsic plasma membrane protein that mediates the active transport and concentration of iodide in the thyroid gland and some extrathyroidal tissues $(8,9)$. GLV-1h68 has also been effective against pancreatic cancer and has demonstrated enhanced tumor kill when combined with the antiangiogenic agent bevacizumab (Avastin; Genentech) (10). 
$h N I S$ is one of several human genes currently being used as a reporter in preclinical studies and has already been used in clinical studies for imaging viral replication in prostate cancer $(11,12)$. hNIS gene transfer via GLV-1h153 allowed infected PANC-1 cells to concentrate carrier-free radionuclide probes such as ${ }^{124} \mathrm{I},{ }^{131} \mathrm{I}$, and ${ }^{99 \mathrm{~m}} \mathrm{Tc}$-pertechnetate $\left({ }^{99} \mathrm{mcO}_{4}\right)$, facilitating deep-tissue imaging of xenografts with PET (6). The noninvasive tracking of virus delivery may provide clinicians with the ability to correlate therapy and efficacy and manage potential viral toxicity and may provide a diagnostic technique to detect tumor origin and the presence of metastases $(11,13)$. Furthermore, GLV-1h153-mediated hNIS expression may facilitate an enhanced tumor therapeutic response when combined with systemic radiotherapy.

This study aimed to determine the feasibility and parameters of serial deep-tissue imaging and long-term noninvasive monitoring of both intratumoral and systemic viral therapy, the response of pancreatic xenografts to GLV-1h153, and factors for successful radiouptake and deep-tissue imaging. The possibility of a correlation between imaging characteristics and tumor volume, optical imaging, and histologic features was also investigated. Furthermore, the potential of combination therapy with carrier-free radioiodine for enhanced pancreatic tumor kill was assessed.

\section{MATERIALS AND METHODS}

\section{Virus and Cell Culture}

African green monkey kidney fibroblast CV-1 cells and human pancreatic ductal carcinoma PANC-1 cells were purchased from American Type Culture Collection and were grown in Dulbecco modified Eagle medium supplemented with $1 \%$ antibiotic-antimycotic solution (Mediatech, Inc.) and 10\% fetal bovine serum (Mediatech, Inc.) at $37^{\circ} \mathrm{C}$ under $5 \% \mathrm{CO}_{2}$. GLV-1h68 was derived from VACV LIVP, as described previously (14). GLV-1h153 was derived from GLV-1h68, as also previously described (6).

\section{PET and CT of Pancreatic Tumor Xenografts in Animal Models}

All animal studies were performed in compliance with all applicable policies, procedures, and regulatory requirements of the Institutional Animal Care and Use Committee, the Research Animal Resource Center of Memorial Sloan-Kettering Cancer Center (MSKCC), and the National Institutes of Health (15). Two groups of animals bearing subcutaneous PANC-1 xenografts on the right hind leg were injected intratumorally $(2$ mice $)$ or intravenously ( 2 mice) with $2 \times 10^{6}$ plaque-forming units (PFU) of GLV-1h153.

The dose of $2 \times 10^{6}$ PFU was chosen using results from a previously published in vivo therapeutic experiment with PANC-1 (6). We elected to investigate both methods of viral administration in this study to assess the potential for virus tumor targeting, therapeutic efficacy, and imaging capability with systemic administration, compared with direct intratumoral injection. Systemic administration of the virus will render it more widely clinically applicable because of reduced invasiveness and the possibility of treating disseminated disease.

One, 2, 3, and 5 wk after viral injection, $9.25 \mathrm{MBq}$ of ${ }^{124} \mathrm{I}$ obtained from the MSKCC radiopharmacy was administered via the tail vein. At $4 \mathrm{~h}$ after radiotracer administration, 3-dimensional listmode data were acquired using an energy window of 350-700 keV and a coincidence timing window of $6 \mathrm{~ns}$. Imaging was performed using a Focus 120 microPET scanner (Concorde Microsystems Inc.) as described previously (6). Four regions of interest (ROIs) were manually drawn on each tumor and averaged for comparison, with the mean injected value \pm SD recorded. A time point of $4 \mathrm{~h}$ after radiotracer injection was chosen due to the balance between strong tumor radiouptake and decreased background uptake. Additionally, at weeks 1 and 2 after virus injection, mice were imaged at $1,4,8,24,48$, and $72 \mathrm{~h}$ after radiotracer injection to obtain time-activity curves. The acquisition time for images obtained at 1 and $4 \mathrm{~h}$ after radiotracer injection was $5 \mathrm{~min}$. At $8 \mathrm{~h}$, the acquisition time was $10 \mathrm{~min}$. At 24, 48, and $72 \mathrm{~h}$ after radiotracer injection, the acquisition time was increased to $20 \mathrm{~min}$ to accommodate radioisotope decay. All images were calibrated so that they were directly comparable.

Moreover, 1 intratumorally and 1 intravenously injected mouse were also imaged with a microCAT II small-animal CT scanner (ImTek Inc.) at $4 \mathrm{~h}$ after radiotracer administration at weeks 1 and 2 , and the obtained images were fused with those obtained with PET that same hour.

\section{Optical Imaging of Pancreatic Tumor Xenografts}

Green fluorescent protein (GFP) expression of tumors infected with GLV-1h153 was visualized at 1,2, 3, and 5 wk after virus injection using the Maestro 2 system (Cambridge Research and Instrumentation). For the bioluminescence imaging, animals were analyzed for the presence of virus-encoded luciferase activity at 1 and $2 \mathrm{wk}$ after virus injection. For this purpose, mice were injected intraperitoneally with a mixture of $5 \mu \mathrm{L}$ of coelenterazine (Sigma; diluted ethanol solution $[0.5 \mathrm{mg} / \mathrm{mL}]$ ) and $95 \mu \mathrm{L}$ of luciferase assay buffer $(0.5 \mathrm{M} \mathrm{NaCl}, 1 \mathrm{mM}$ ethylenediaminetetraacetic acid, and $0.1 \mathrm{M}$ potassium phosphate [pH 7.4]). Bioluminescence was then measured with an IVIS 100 imaging system (Xenogen) by collecting photons for $1 \mathrm{~min}$ from dorsal views of the animals and analyzed using the Living Image 2.5 software (Xenogen). These different imaging modalities were used to guide the choice of time points for the PET experiments and to confirm virus colonization of tumors.

\section{Histologic Confirmation of GLV-1h153 Infection of Pancreatic Tumor Xenografts}

Five weeks after viral injection and after imaging, all mice were sacrificed and tumors harvested. The immunohistochemistry detection of anti-VACV A27 L antigen antibodies was performed at the Molecular Cytology Core Facility of MSKCC using a Discovery XT processor (Ventana Medical Systems). Slides were incubated with polyclonal antibody produced in rabbits against synthetic peptide AKKIDVQTGRRPYE (the C terminal of A27 L vaccinia protein) (custom-made by GenScript Corp.) at a dilution of 1:1,000 for $5 \mathrm{~h}$, followed by a 60-min incubation with biotinylated goat antirabbit IgG (PK6101; Vector Labs) in a 1:200 dilution. A secondary antibody blocker (blocker D), streptavidin-HRP, and a diaminobenzidine detection kit (Ventana Medical Systems) were used according to manufacturer instructions. Slides were counterstained with hematoxylin (760-2021; Ventana Medical Systems) and bluing reagent (760-2037; Ventana Medical Systems) at 4 min before mounting, also according to manufacturer instructions. Negative IgG controls were used for comparison. 


\section{Calculations of Absorbed Radioiodine Doses in Virus-Colonized Pancreatic Tumors}

To calculate absorbed doses in virally treated tumors, 4 ROIs were manually drawn on imaged tumors and averaged, with the mean injected value $\pm \mathrm{SD}$ recorded. The ${ }^{124} \mathrm{I}$ image-derived time-activity concentration data were corrected for radioactive decay to the time of injection and fit to exponential functions using least-squares regression $(16,17) .{ }^{131} \mathrm{I}$ cumulated activity concentrations (in $\mathrm{MBq}-\mathrm{h} / \mathrm{g} / \mathrm{MBq}$ of ${ }^{131} \mathrm{I}$ administered) were then calculated using Excel (Microsoft Corp.) by integrating the fitted time-activity functions, incorporating the physical decay constant of ${ }^{131} \mathrm{I}(0.0036 / \mathrm{h})$. Mean tumor-absorbed doses (Gy/MBq of ${ }^{131} \mathrm{I}$ administered) were calculated assuming complete local absorption of the ${ }^{131} \mathrm{I} \beta$-rays $(0.109 \mathrm{~g}-\mathrm{Gy} / \mathrm{MBq}-\mathrm{h}[0.405$ $\mathrm{g}-\mathrm{rad} / \mu \mathrm{Ci}-\mathrm{h}])$ and ignoring the small contribution of the ${ }^{131} \mathrm{I} \gamma$-rays.

\section{Autoradiography}

Three mice bearing PANC-1 xenografts on the right hind leg were injected intratumorally with $2 \times 10^{7}$ PFU of GLV-1h153 (2 mice) or phosphate-buffered saline (PBS) (1 mouse). Two days after injection, $33.3 \mathrm{MBq}$ of ${ }^{99} \mathrm{~m}^{\mathrm{m}} \mathrm{T}_{4}$ obtained from MSKCC's radiopharmacy were administered via the tail vein. Ventral and dorsal planar images of the in vivo distribution of ${ }^{99} \mathrm{mcO}_{4}$ were simultaneously acquired using the dual-detector $\gamma$-camera subsystem of the XSPECT smallanimal SPECT/CT system (Gamma Medica) at $0.5 \mathrm{~h}$ after radiotracer administration. The detectors were fitted with low-energy, high-resolution parallel-hole collimators, and images were acquired over 10 min using a $140-\mathrm{keV}+10 \%{ }^{99} \mathrm{TcO}_{4}$ photopeak energy window and a $56 \times 56$ image matrix (pixel size, $2.2 \times 2.2 \mathrm{~mm}$ ). A higher virus dose was used in this experiment because small amounts of virus were injected at different areas of the tumor to create individual radiouptake hot spots in order to elucidate possible factors involved in uptake, compared with areas without enhanced radiouptake.

After imaging, the fluorescent dye Hoechst 33342 trihydrochloride (Sigma) $(40 \mathrm{mg} / \mathrm{kg} ; 1 \mathrm{mg}$ in $100 \mu \mathrm{L}$ of physiologic saline) was injected via the tail vein. Five minutes after dye injection, the animals were sacrificed and tumors excised, embedded in mounting medium (O.C.T. Compound; Sakura Finetek), and frozen on dry ice. Sets of contiguous frozen tissue sections were cut at a $6-\mu \mathrm{m}$ thickness on a Microm HM500 cryostat microtome (Microm International $\mathrm{GmbH}$ ) and collected on glass microscope slides. Digital autoradiography was then performed on tissue sections. Sections were placed in a film cassette against a phosphor imaging plate (Fujifilm BAS-MS2325; Fuji Photo Film). Phosphor plates were read out at a resolution of $50 \times 50 \mu \mathrm{m}$ on a BAS-1800II BioImaging Analyzer (Fujifilm Medical Systems). Digital images of the distributions of GFP and Hoechst 33342 in tumor sections were acquired at $\times 100$ magnification using a fluorescence microscope (Diaphot 300; Nikon) equipped with a computer-controlled motorized stage and digital Coolsnap EZ camera (Photometrics) for image capture. After acquisition of fluorescence images, tumor sections were stained with hematoxylin and eosin (H\&E) and imaged by light microscopy. Composite images of whole tumor sections were obtained by stitching individual microscopic images using Image-Pro software (Image-Pro Plus, version 7.0; MediaCybernetics). All images were saved in 8-bit format and manually coregistered using Adobe Photoshop (version 7.0; Adobe Systems).

\section{Combination Therapy of GLV-1h153 and ${ }^{131}$ I}

For the radiotherapeutic experiment, PANC-1 xenografts were established in the right hind leg in 5 groups of mice and systemically treated with GLV-1h153 plus $37 \mathrm{MBq}$ of ${ }^{131} \mathrm{I}$ (7 mice), GLV-1h153 plus $92.5 \mathrm{MBq}$ of ${ }^{131} \mathrm{I}$ (8 mice), GLV-1h153 alone (5 mice), $92.5 \mathrm{MBq}$ of ${ }^{131} \mathrm{I}$ alone (4 mice), or PBS control (4 mice). Two weeks after intravenous injection of $1 \times 10^{5} \mathrm{PFU}$ of GLV-1h153 or PBS, treated groups were administered $37 \mathrm{MBq}$ or $92.5 \mathrm{MBq}$ of ${ }^{131} \mathrm{I}$ by a single intravenous injection. Tumor volumes were measured before the initial administration of virus or PBS and weekly thereafter using the formula ([height $-5 \mathrm{~mm}] \times$ length $\times$ width). All mice were followed up until $6 \mathrm{wk}$ after virus injection. ${ }^{131} \mathrm{I}$ was obtained from MSKCC's radiopharmacy. The lower dose of 1E5 PFU of virus was chosen to investigate whether combination therapy may work better than either virus or radiotherapy alone. Using lower doses of virus and lower doses of radiotherapeutics has the potential to lessen systemic toxicity and side effects associated with either treatment.

\section{Statistical Analysis}

For treatment experiments, the GraphPad Prism program (version 5.0; GraphPad Software) was used for data handling and analysis. The significance between therapy groups was determined via 2-way ANOVA with Bonferroni adjustment. A $P$ value less than 0.05 was considered significant.

\section{RESULTS}

\section{GLV-1h153 Facilitates Serial Long-Term Monitoring of Systemic and Intratumoral Viral Therapy}

PET detected virus replication in tumors as late as $5 \mathrm{wk}$ after injection (Fig. 1A). hNIS-mediated radiouptake peaked at $2 \mathrm{wk}$ after virus injection and gradually declined by weeks 3 and 5 in all mice except mouse 3 . This decline was despite a persistent strong GFP signal at 5 wk after injection (Fig. 1B).

\section{PET Signal Pattern Correlates with Antitumor Response to Viral Therapy}

When comparing PET signal intensity with tumor growth patterns over $5 \mathrm{wk}$, tumor radiosignal correlated with initial tumor growth, growth retardation, and regression. The PET signal remained strong during the first $2 \mathrm{wk}$ after the injection of the virus, reflecting initial tumor growth. However, when tumor growth began entering a plateau phase, the PET signal began to decrease and almost disappeared in most mice by week 5 , when tumors entered the regression phase. Interestingly, compared with those of mouse 1 , mouse 2 , and mouse 4 , the tumor harbored by mouse 3 regressed more slowly and maintained a relatively strong PET signal at 5 wk (Fig. 1C).

\section{GLV-1h153-Mediated ${ }^{124}$ I PET Signal Persists Even at 24 Hours After Radiotracer Injection}

PET readily detected virus replication in tumors as late as $24 \mathrm{~h}$ after radiotracer injection at both week 1 and week 2 after virus injection (Fig. 2A). To calculate absorbed doses in tumors, time-activity concentration data were generated from serial images using ROIs. At 1 wk after virus injection, ${ }^{124}$ I uptake was maximal at $1 \mathrm{~h}$ after radiotracer injection at an average of $8.39 \pm 1.23$ percentage injected dose per gram $(\% \mathrm{ID} / \mathrm{g})$ for the intravenous group and $10.81 \pm 1.10 \% \mathrm{ID} / \mathrm{g}$ for the intratumoral group. Uptake exponentially declined over $72 \mathrm{~h}$ but was retained as late as $24 \mathrm{~h}$ and even at $48 \mathrm{~h}$ after injection. Interestingly, image and time-activity 


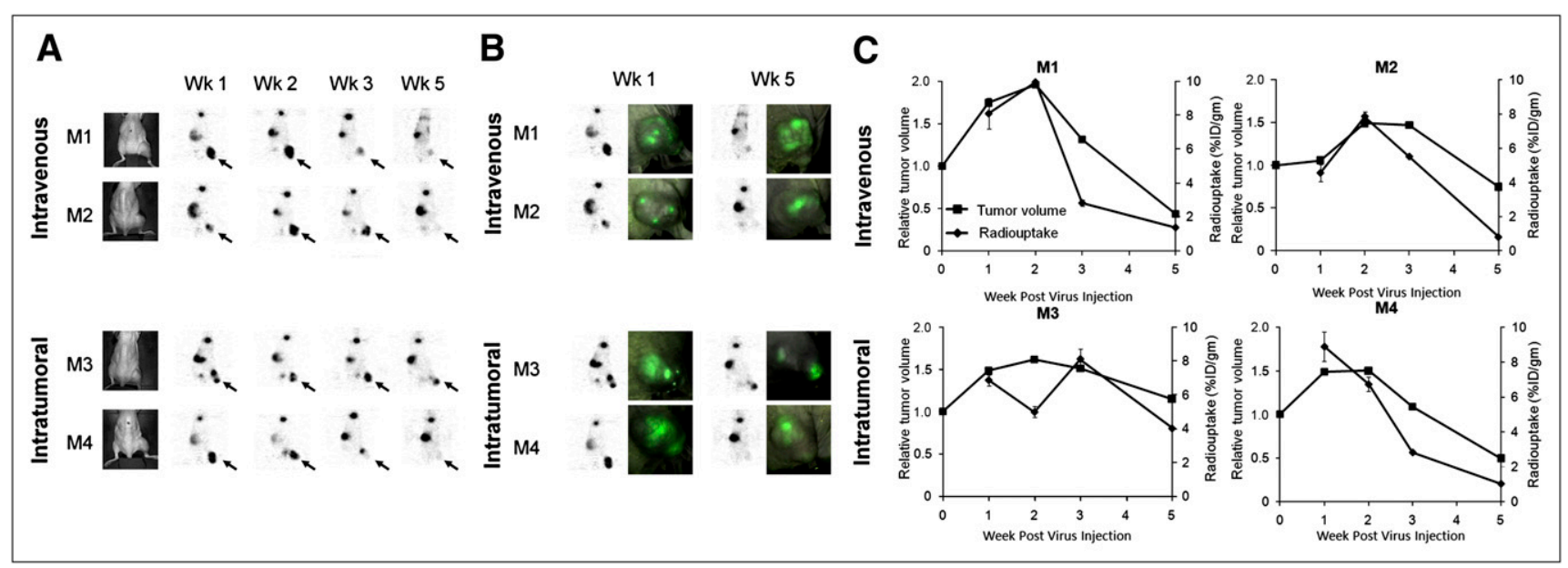

FIGURE 1. GLV-1h153 facilitates serial monitoring of systemic and intratumoral viral therapy. (A) hNIS-mediated enhanced radiouptake in GLV-1h153-infected tumors was serially monitored, being strongest at weeks 1 and 2 after virus injection and declining gradually by weeks 3 and 5 except for M3 (shown with arrows). Uptake was also noted in bladder because of radiotracer clearance and in thyroid and stomach because of intrinsic NIS expression. (B) PET signal declined despite persistent strong GFP expression at 5 wk after virus injection. (C) PET signal appeared to reflect tumor response to therapy and remained strong during initial tumor growth. However, when tumor growth began entering plateau or retardation phase, PET signal began to decrease and almost disappear by week 5 , when tumors entered regression phase. $M 1, M 2, M 3$, and M4 = mouse 1 , mouse 2 , mouse 3 , and mouse 4, respectively.

data were similar in week 2 , with a slightly increased maximal uptake of $10.14 \pm 1.43 \% \mathrm{ID} / \mathrm{g}$ for the intravenous group and a decreased maximal uptake of $8.05 \pm 0.01 \%$ $\mathrm{ID} / \mathrm{g}$ for the intratumoral group at $1 \mathrm{~h}$ after radiotracer injection (Fig. 2B).

\section{GLV-1h153 Tumor Infection Is Confirmed Via GFP, Bioluminescence, and PET/CT}

GLV-1h153 colonization of tumors facilitating hNISmediated radiouptake was also confirmed via GFP, bioluminescence, and PET/CT at 1 and $2 \mathrm{wk}$ after virus injection in both the intravenous and the intratumoral groups. These reporter genes were detectable, and the fusion of PET and CT images correlated the enhanced signals anatomically to the tumor, stomach, bladder, and thyroid (Fig. 3).

\section{Histology of GLV-1h153-Treated Tumors Is Examined at 5 Wk After Injection}

Histologic examination of serially imaged tumors confirmed wide areas of necrosis and inflammatory infiltrate at $5 \mathrm{wk}$ after virus injection. Necrotic areas were more homogeneous in the intravenously treated group, whereas islands of necrosis surrounded by inflammatory infiltrate were seen in the intratumoral group, reflecting a viral route of administration. Interestingly, despite careful examination of tumor sections with a pathologist, no viable cells were detected histologically in any tumors except that of mouse 3 , which retained some viable tumor proliferation activity and, as previously noted, was the only mouse to retain a relatively strong PET signal (Fig. 1C). The presence of virus in tumors treated with GLV-1h153 was confirmed, and tumor tissue stained positively for vaccinia $\mathrm{A} 27 \mathrm{~L}$ antigen, yielding a brownish precipitate, compared with the pink-purple H\&E background seen with uninfected areas and control tumors (Fig. 4).

\section{hNIS-Mediated Radiotracer Uptake Requires Presence of GLV-1h153, Viable Tissue, and Adequate Blood Flow}

To determine what conditions are needed for adequate GLV-1h153-mediated radiouptake and retention in tumors, autoradiography was performed with ${ }^{99} \mathrm{~m}_{\mathrm{TcO}}$-mediated $\gamma$-scanning. Mice were intravenously injected with Hoechst dye to stain for blood flow, and tumors were harvested and sectioned to determine regions of uptake. The short viral treatment duration and localized intratumoral administration led to hot spots of ${ }^{99} \mathrm{TcO}_{4}$ uptake in the tumor, which, when compared with adjacent slices, revealed the need for both GLV-1h153 presence (reflected by GFP expression) and adequate blood flow (shown with Hoechst staining). No uptake was noted in control tumors, despite viable tissue and adequate blood flow (Fig. 5A). Looking more closely at merged images, hot spots again corresponded to areas with GFP expression (thus virus) and Hoechst staining (thus blood flow), whereas areas that lacked GFP and areas noted to be necrotic on H\&E staining with lack of blood flow did not support uptake (Fig. 5B).

\section{GLV-1h153 Has Potential to Deliver Targeted Therapeutic Doses of Radioiodine to Tumors}

To calculate absorbed doses in tumors, the image-derived time-activity concentration data were corrected for radioactive decay to the time of injection and fit to exponential functions using least-squares regression. The ${ }^{131} \mathrm{I}$ cumulated activity concentrations (in $\mathrm{MBq}-\mathrm{h} / \mathrm{g} / \mathrm{MBq}$ of ${ }^{131} \mathrm{I}$ administered) were then calculated by integrating the fitted time-activity functions, incorporating the physical decay constant of ${ }^{131} \mathrm{I}$. For the intravenous group, the average absorbed doses were $154.23 \pm 48.35 \mathrm{~Gy} / \mathrm{MBq}$ at week 1 and $190.39 \pm 8.40 \mathrm{~Gy} / \mathrm{MBq}$ at week 2 after virus injection. 


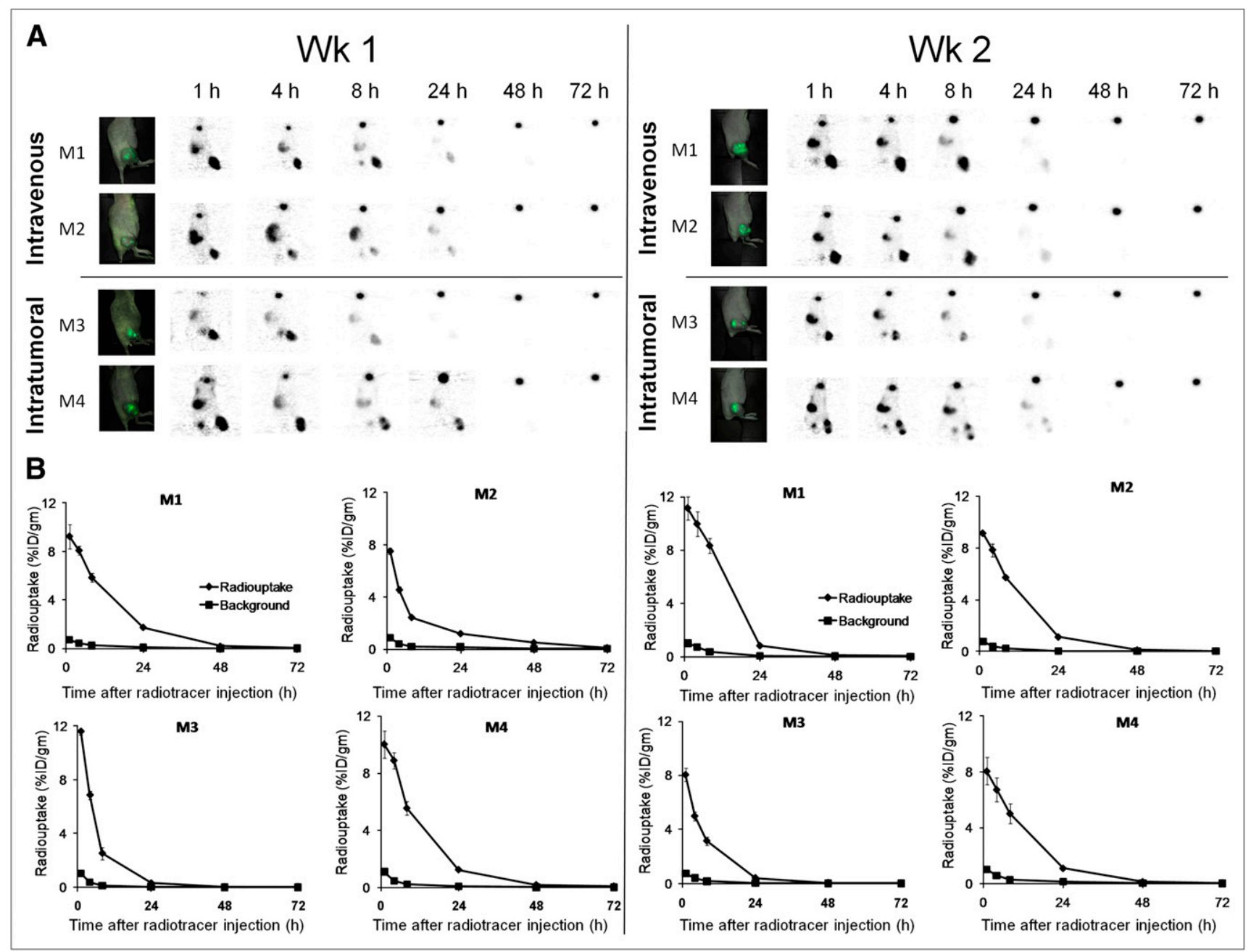

FIGURE 2. GLV-1h153 mediates radiotracer retention for at least $24 \mathrm{~h}$ after administration. (A) ${ }^{124}$ I PET image signal was strongest at $1 \mathrm{~h}$ after radiotracer injection and gradually declined over 72 h. (B) Time-activity curves were generated from PET images using ROls manually drawn over tumors. Radiouptake mediated and enhanced by hNIS in GLV-1h153-infected tumors could be serially monitored and measured, with quantitative uptake being strongest at $1 \mathrm{~h}$ after radiotracer injection and exponentially declining over $72 \mathrm{~h}$. Maximum radiouptake ranged from approximately $8-11 \% \mathrm{ID} / \mathrm{g}$ for intravenous and intratumoral groups at both week 1 and week 2 after virus injection, highlighting tumor-homing ability of GLV-1h153. M1, M2, M3, and M4 = mouse 1, mouse 2, mouse 3, and mouse 4, respectively.

For the intratumoral group, the average absorbed doses were $143.20 \pm 38.70 \mathrm{~Gy} / \mathrm{MBq}$ at week 1 and $130.57 \pm 35.32 \mathrm{~Gy} /$ $\mathrm{MBq}$ at week 2. The absorbed dose at week 1 after virus injection appeared higher in the intravenous group, despite a lower maximal radiouptake, signifying longer retention in the tumor. Table 1 shows a summary of uptake, effective half-lives, and absorbed calculated doses in tumors.

\section{Combining GLV-1h153 and Radioiodine Mediates Greater Therapeutic Efficacy}

Whereas the PBS, GLV-1h153 alone, and ${ }^{131} \mathrm{I}$ alone groups of treated mice bearing PANC-1 xenografts continued to experience rapid tumor growth, the groups treated with GLV-1h153 plus ${ }^{131}$ I experienced a retardation and even regression of tumor volume (Fig. 6). This effect was statistically significant $(P<0.01$ by day 42 when compared with GLV-1h153 or ${ }^{131}$ I alone).

\section{DISCUSSION}

Oncolytic VACVs have shown promise for the treatment of solid tumors, including pancreatic cancer, and future clinical studies with VACV may benefit from the ability to noninvasively and serially identify sites of viral targeting in addition to measuring the level of viral infection for correlation with safety, efficacy, and toxicity $(11,13,18)$. Such real-time tracking would also provide useful viral dose and administration scheduling information for the optimization of therapy and would obviate multiple and repeated tissue biopsies.

We have previously reported on the visualization of tumors and metastases in live animals using VACV-encoding light-emitting proteins with optical surface imaging (19). We then explored the possibility of deep-tissue modalities and reported on the construction of a vaccinia virus carrying the human norepinephrine transporter (20). However, a limitation 
FIGURE 3. Several GLV-1h153 reporter genes are detectable. GFP, bioluminescence, and hNIS signal could be detected in GLV1h153-infected tumors. Fusion of PET and CT images correlated hNIS-mediated uptake signal anatomically to location of thyroid, stomach, bladder, and tumor, whereas GFP and Ruc-mediated bioluminescence signals located only to tumor. M2 and M4 = mouse 2 and mouse 4, respectively. $W 1$ and $W 2=$ week 1 and week 2 , respectively.

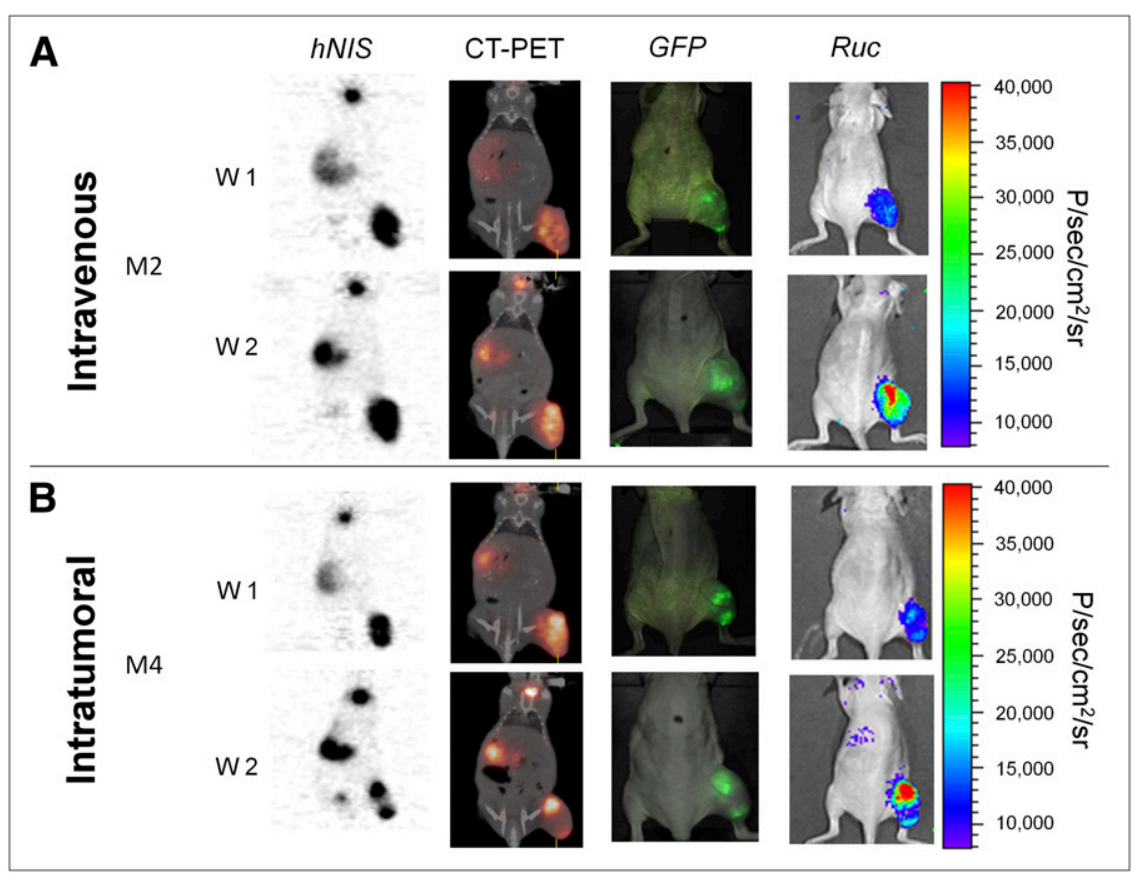

of the human norepinephrine transporter is the requirement for the carrier metaiodobenzylguanidine for the uptake of radioprobes. GLV-1h153, an oncolytic VACV encoding for hNIS, has also been successful for deep-tissue imaging and the treatment and localization of viral replication in tumors (6). hNIS, an intrinsic plasma membrane protein that is unlike human norepinephrine transporter, mediates the active transport and concentration of several carrier-free radionuclide probes that have long been approved for human use, facilitating both deep-tissue imaging of viral therapy and potential targeted radiotherapy $(9,21,22)$.
Here we have shown, for the first time to our knowledge, the feasibility, parameters, and determining factors of serial long-term monitoring of both systemically and intratumorally administered GLV-1h153 for the treatment of pancreatic cancer using ${ }^{124} \mathrm{I}$ PET and ${ }^{99} \mathrm{mcO}_{4} \gamma$-scintigraphy and the potential for combination therapy with systemic radiotherapeutic ${ }^{131} \mathrm{I}$. We have also explored determining factors for successful radiouptake by tumors and insight provided by serial long-term imaging. The time points chosen for serial long-term PET of viral therapy were based on results from our foundation paper showing the
FIGURE 4. Histologic examination of virus-treated tumors at $5 \mathrm{wk}$ after injection reveals ability of GLV-1h153 to provide insight into tumor biologic activity. (A) Histologic examination of imaged tumors at $5 \mathrm{wk}$ after virus injection showed wide areas of necrosis and inflammatory infiltrate. (B) No viable cells were detected histologically in mouse tumors except in mouse 3 , which retained some tumor proliferation activity, as seen at $\times 400$ magnification. Presence of GLV-1h153 in tumors was confirmed histologically, shown here with the brownish precipitate against vaccinia $A 27 \mathrm{~L}$ antigen in intravenous and intratumoral groups ( $A$ and $B$, respectively). Bars represent $5,000 \mu \mathrm{m}$. $\mathrm{M} 1, \mathrm{M} 2, \mathrm{M} 3$, and $\mathrm{M} 4=$ mouse 1 , mouse 2 , mouse 3 , and mouse 4 , respectively.

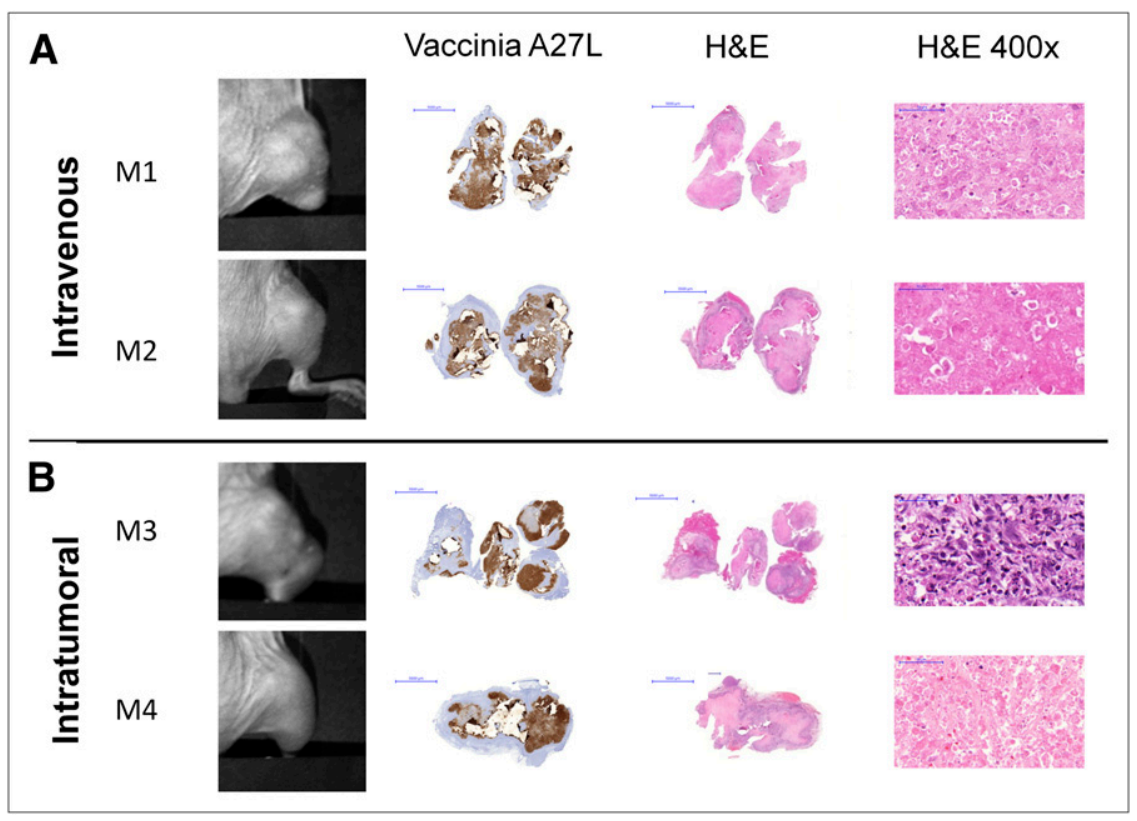




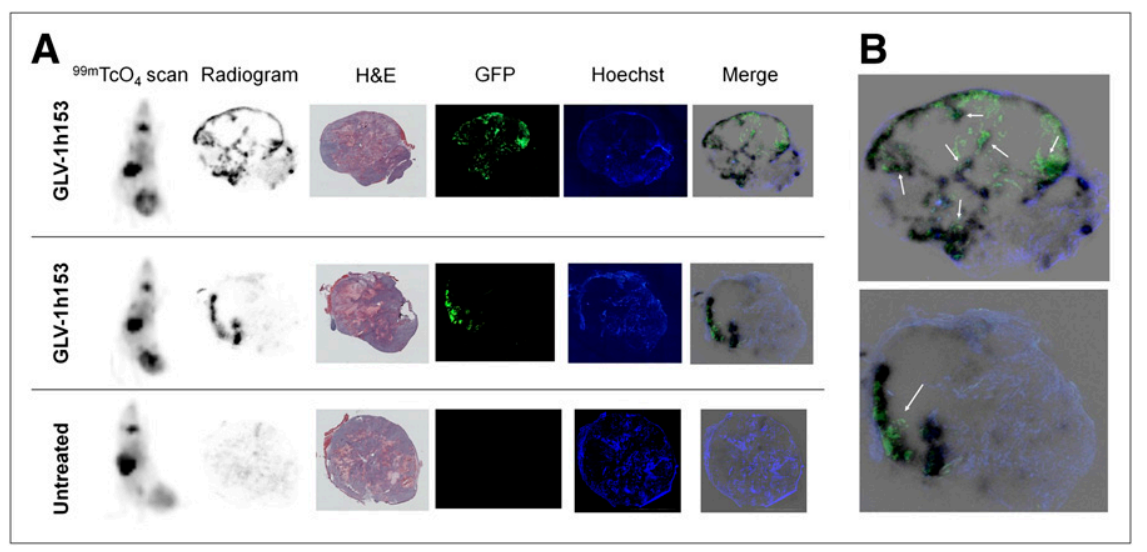

FIGURE 5. Autoradiography of tumors injected with GLV-1h153. (A) Tissue radio-

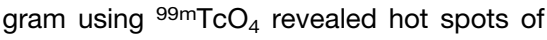
radiouptake in GLV-1h153 tumors correlating with expression of GFP and Hoechst staining, revealing requirement for both presence of GLV-1h153 infection and adequate blood flow to deliver radiotracer. Areas that lacked GFP and thus virus and areas noted on H\&E staining to be necrotic and lacking blood flow did not support uptake. No uptake was noted in untreated control tumors despite adequate blood flow. (B) Enlarged merged sections correlated hot spots with GFP and Hoechst dye (shown with arrows). therapeutic effect of viral treatment on PANC-1 tumors over a period of $5 \mathrm{wk}(6)$.

In contrast to several studies with other oncolytic viruses, such as measles and adenoviruses, which had signals lasting approximately $5 \mathrm{~d}$ after virus injection, PET readily detected virus replication in pancreatic tumor xenografts as late as 5 wk after GLV-1h153 injection (23-25). hNIS-mediated radiouptake with GLV-1h153 seemed to peak at 2 wk and gradually declined by weeks 3 and 5 in both the intratumorally and the intravenously injected groups. This finding is interesting, because although the signal timing pattern in the intravenous group was similar to what others have found with VSV (51)NIS and MV-NIS $(26,27)$, the intratumoral group had a more prolonged uptake enhancement, possibly because of the early replication and rapid spread of vaccinia, facilitating greater hNIS expression throughout the tumor before cell lysis and death occurs. This principle can be built on in future experiments and potentially applied to clinical practice as viral delivery mechanisms are chosen. It is difficult to compare uptake values achieved with other oncolytic viruses, because dose effects may differ and are unlikely to be directly comparable. A decrease in PET signal enhancement with GLV1h153 occurred despite persistent strong GFP expression by 5 wk after treatment, suggesting that GFP does not provide insight into tumor response. Several GLV-1h153 reporter genes were detectable, highlighting the multifunctional aspects of the biotechnology, and the fusion of PET and CT images correlated the NIS-mediated signals anatomically to the location of the tumor, as well as to the stomach and thyroid (because of intrinsic NIS expression) and the bladder (because of radiotracer clearance).

When PET signal intensity is compared with tumor growth patterns in mice over $5 \mathrm{wk}$, enhanced radiosignal appears to correlate with the phases of initial tumor growth, retardation, and eventual regression. The PET signal remained strong during the first $2 \mathrm{wk}$ after injection of virus, reflecting initial tumor growth. However, when tumor growth began entering a retardation phase, PET signal began to decrease and almost disappear by week 5, when tumors entered the regression phase. To our knowledge, correlation of hNIS-mediated uptake and signal patterns was not explored in any other paper investigating oncolytic viruses encoding hNIS. It is known that some delay occurs before an actual difference in tumor size is noted, even after cell death or necrosis has occurred after viral treatment. The only exception was a single intratumorally treated mouse (mouse 3); this mouse demonstrated a persistently strong signal up to $5 \mathrm{wk}$ after GLV1 h153 injection, which coincided with increased tumor regression time. These data suggest that the hNIS reporter gene, unlike GFP, is able to provide insight into the biologic activity of tumor cells and determine whether cell death or necrosis is occurring. Nonviability of cells and loss of cell membrane integrity is likely to affect the functionality of the hNIS protein, as shown in previous cell culture radiouptake experiments (6). The GFP reporter protein, however, simply suggests viral replication and, once produced, seems to persist despite nonviability of tumor cells. This finding has important implications for future noninvasive reporting of response to viral therapy in clinical practice.

These results were supported by histologic analysis of serially imaged tumors, which confirmed wide areas of necrosis and inflammatory infiltrate. Tumor cell death and necrosis may explain the lack of PET signal in the other imaged mice at $5 \mathrm{wk}$ after virus injection and the persistence of signal in mouse 3, which retained viable tumor proliferation activity. However, staining for the A27VACV antigen revealed viral persistence in the tumors, although this may not necessarily reflect persistence of live virus but may be explained by the fact that virus particles can persist in the tumor microenvironment despite nonviability of actual tumor cells. Similar to the GFP signal, the persistence of virus does not give insight into tumor cell response to viral therapy.

Time-activity concentration data over $72 \mathrm{~h}$ after radiotracer injection generated from imaged mice in both the intravenous and the intratumoral groups revealed similar maximal ${ }^{124}$ I uptake at $1 \mathrm{~h}$ after radiotracer injection and $1 \mathrm{wk}$ after virus injection, highlighting the tumor-homing ability of the virus even when injected systemically. Uptake declined over $72 \mathrm{~h}$ in a classic exponential manner but could still be detected in tumors at 24 and even $48 \mathrm{~h}$ after radiotracer injection. Uptake appears to be similar to that 


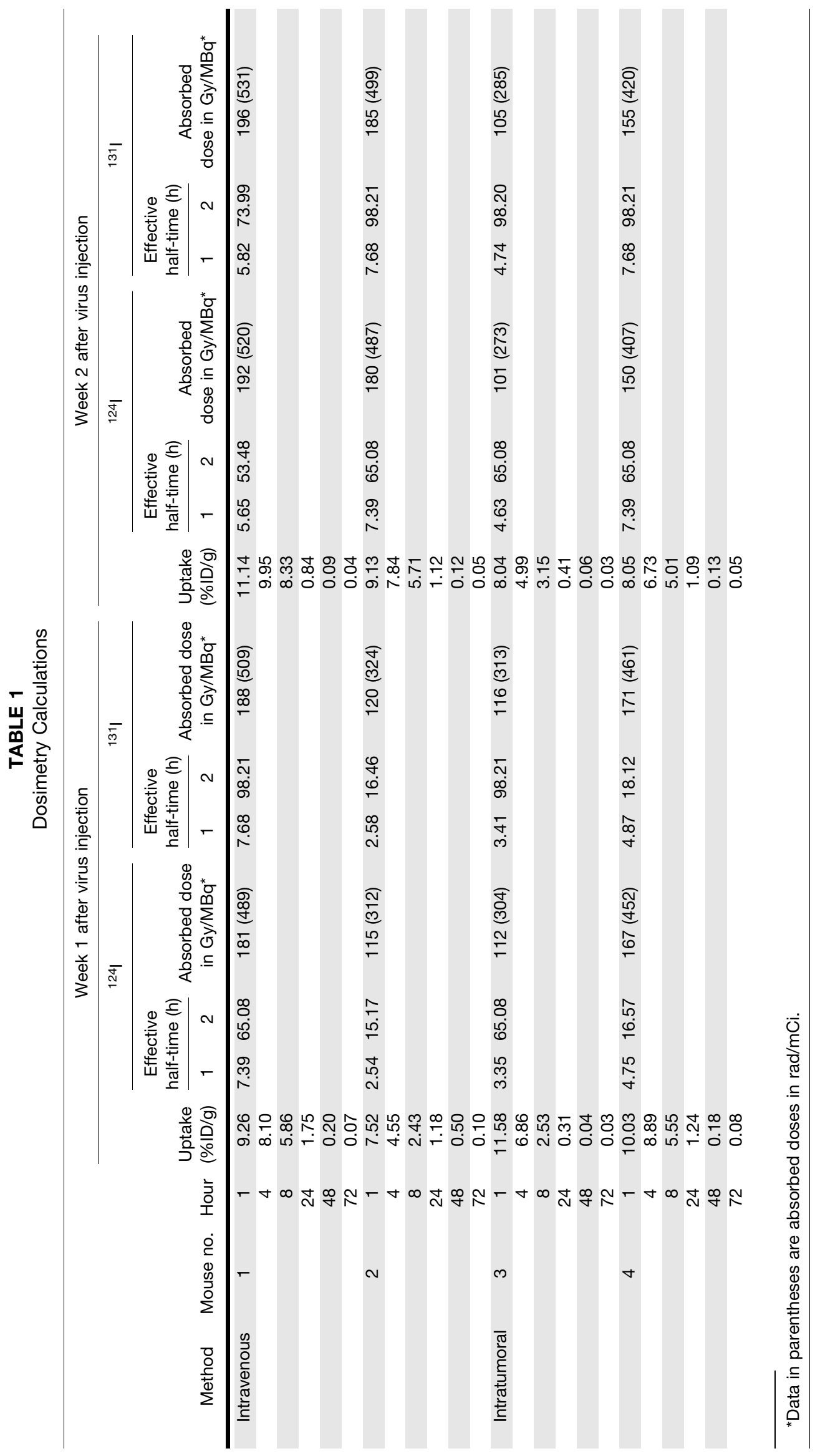

1940 The Journal of Nuclear Medicine • Vol. 53 • No. 12 • December 2012 


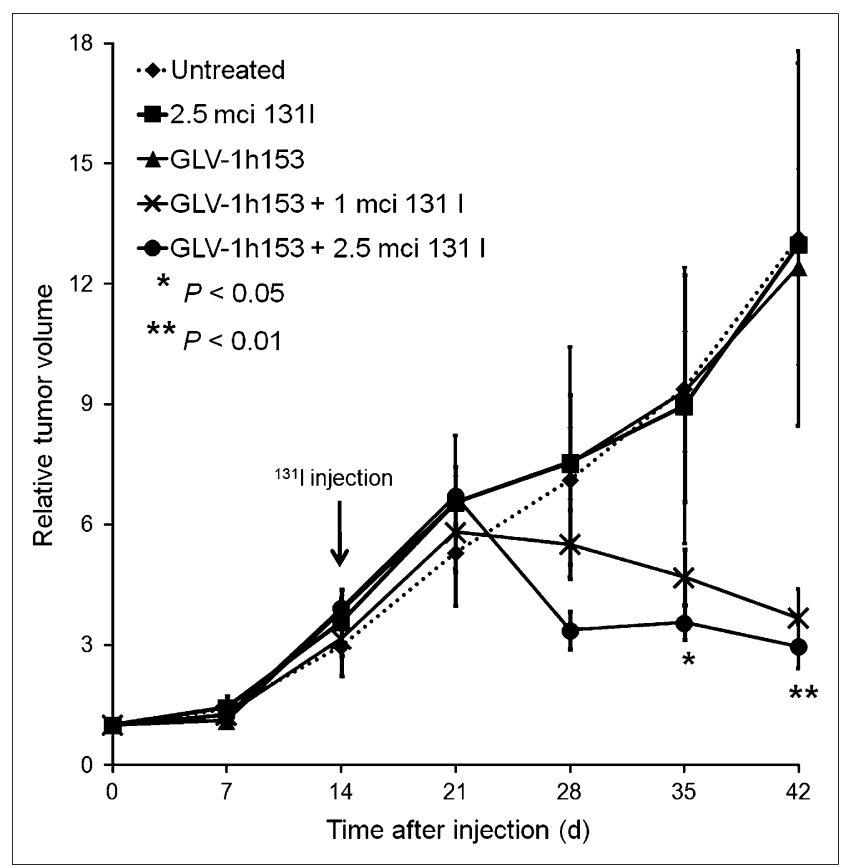

FIGURE 6. Combination therapy with GLV-1h153 and ${ }^{131}$ I mediates enhanced tumor kill. Although groups of mice treated with PBS, GLV-1h153 alone, and ${ }^{131}$ I alone continued to experience rapid tumor growth in radiovirotherapeutic experiment, mice treated with GLV-1h153 plus ${ }^{131}$ I experienced retardation and even regression of tumor volume. Statistical analysis was performed using 1 -way ANOVA. ${ }^{* *} P<0.01$ for comparison of untreated control, GLV-1h153, or ${ }^{131}{ }^{1}$ groups alone with GLV-1h153 plus ${ }^{131}$ I groups. ${ }^{\star} P<0.05$ for comparison of untreated control, GLV-1h153, or ${ }^{131}$ I groups alone with GLV-1h153 plus ${ }^{131}$ I groups.

mediated by studies with MV-NIS and some adenoviruses but was also lower than some reported uptake values, especially when diet was supplemented with thyroxine and when iodine intake was low $(27,28)$. This observation highlights the potential of maximizing radioiodine uptake in tumors by suppressing uptake in the stomach and thyroid because of intrinsic NIS expression.

Absorbed doses from image-derived time-activity concentration data of GLV-1h153-infected PANC-1 tumors were then calculated assuming ${ }^{131} \mathrm{I}$ treatment. The absorbed dose at week 1 after virus injection was higher in the intravenous group, despite a lower maximal radiouptake, signifying longer retention in the tumor. Further, the accumulated dose was at a potentially therapeutic level and was similar to results obtained with MV-NIS for the treatment of prostate cancer (26). It is unclear how radioiodine is trapped within virus-transduced cancer cells. hNIS increases the uptake of radioiodine but does not maintain retention $(9,29)$. One possible explanation is that hNIS may continually take up radioiodine that is being circulated in the vasculature at the same time that radioiodine is flowing out from the virus-transduced cells. The decrease in radiouptake over time is likely the result of a combination of radioiodine cellular efflux and a decrease in circulating radioiodine because of decay and renal clearance.
In the autoradiography experiment, tumor areas that lacked GFP expression-and thus virus colonizationand areas noted to be necrotic on H\&E staining with lack of Hoechst staining - and thus blood flow-did not support uptake on scintigraphy or autoradiography. Necrosis could have been virally mediated or intrinsic because of tumor enlargement. However, this may explain the loss of signal noted with serial monitoring of infected tumors, because necrotic areas occurring secondary to viral treatment lacked blood flow and, likely, functional cell membranes and thus were unlikely to facilitate hNIS-mediated uptake in tumors. Neither GFP nor radiouptake was noted in uninfected control tumors, despite adequate blood flow and viable tissue.

Therefore, it was recognized that the dose of virus and timing of administration of radioiodine for combination treatment of pancreatic tumor xenografts was of primary importance. Hence, with a lower treatment dose of virus and an early administration of radioiodine, combination therapy of GLV-1h153 and ${ }^{131}$ I revealed a statistically significantly enhanced tumor kill when compared with GLV-1h153 or ${ }^{131} \mathrm{I}$ alone by day 42 after virus injection. Lower doses of virus were used to show this additive effect, because tumor kill with GLV-1h153 alone has already been shown effective at the previously selected dose of $2 \times 10^{6} \mathrm{PFU}(6)$, and thus a potential difference would be more difficult to detect if GLV-1h153 was combined with radiotherapy at this same dose. Using lower doses of virus and lower doses of radiotherapeutics has the potential to lessen systemic toxicity and side effects associated with either treatment. Furthermore, combination therapies may lessen the chance that tumor resistance to treatments may develop. These results were similar to those of other studies showing the potential of enhanced treatment efficacy by combining ${ }^{131}$ I treatment with hNIS-encoding oncolytic viruses in several tumor models (26-28,30-32). Further work is required to assess the optimum doses of virus to be combined with radiotherapy to obtain an additive or synergistic therapeutic effect. Moreover, a disadvantage of using hNIS is the potential for a large radiation dose to the thyroid and stomach due to endogenous hNIS expression. Possible strategies to overcome this disadvantage include pretreatment with thyroxine supplementation and a low-iodine diet to decrease endogenous hNIS expression.

\section{CONCLUSION}

The GLV-1h153 vaccinia construct is a promising candidate for future clinical studies combining deep-tissue imaging, oncolytic virotherapy, and systemic radiotherapy. GLV1h153-treated pancreatic tumors were successfully serially imaged and treated with the clinically approved radiopharmaceutical ${ }^{124} \mathrm{I}$ and PET and with ${ }^{99} \mathrm{~m} \mathrm{TcO}_{4}$-mediated $\gamma$-scintigraphy. Furthermore, radioiodine dose and retention time in infected tumors accumulated at potentially therapeutic levels, and the combination of GLV-1h153 and ${ }^{131}$ I was promising for more rapid and potent tumor regression. Further investigation is required into the effect of viral dose, tumor size, and metastatic disease on imaging capability 
and the potential for targeted radiotherapy with this novel viral technology.

\section{DISCLOSURE STATEMENT}

The costs of publication of this article were defrayed in part by the payment of page charges. Therefore, and solely to indicate this fact, this article is hereby marked "advertisement" in accordance with 18 USC section 1734.

\section{ACKNOWLEDGMENTS}

We acknowledge the support of William $\mathrm{H}$. and Alice Goodwin and the Commonwealth Foundation for Cancer Research, and the Experimental Therapeutics Center of Memorial Sloan-Kettering Cancer Center. The funders had no role in study design, data collection and analysis, decision to publish, or preparation of the manuscript. We also thank Jenifer Levin and Raia Mohammed for their skillful editing of the manuscript. Appreciation is extended to MSKCC's radiopharmacy for kindly providing radioisotopes. Thanks are also given to Jason Aguilar, Terry Trevino, Melody Fells, and Megan Reese for excellent technical assistance. Gratitude is extended to Dr. Victor Andrade for help with histologic interpretation. Nanhai G. Chen, Qian Zhang, Yong A. Yu, Richard J. Aguilar, and Aladar A. Szalay are employees of Genelux Corporation. No other potential conflict of interest relevant to this article was reported.

\section{REFERENCES}

1. Galasso D, Carnuccio A, Larghi A. Pancreatic cancer: diagnosis and endoscopic staging. Eur Rev Med Pharmacol Sci. 2010;14:375-385.

2. Squadroni M, Fazio N. Chemotherapy in pancreatic adenocarcinoma. Eur Rev Med Pharmacol Sci. 2010;14:386-394.

3. Vähä-Koskela MJ, Heikkila JE, Hinkkanen AE. Oncolytic viruses in cancer therapy. Cancer Lett. 2007;254:178-216.

4. Chen NG, Szalay AA. Oncolytic vaccinia virus: a theranostic agent for cancer. Future Virol. 2010;5:763-784.

5. Thorne SH, Kirn DH. Future directions for the field of oncolytic virotherapy: a perspective on the use of vaccinia virus. Expert Opin Biol Ther. 2004;4:1307-1321.

6. Haddad D, Chen NG, Zhang Q, et al. Insertion of the human sodium iodide symporter to facilitate deep tissue imaging does not alter oncolytic or replication capability of a novel vaccinia virus [abstract]. J Transl Med. 2011;9:36.

7. Gholami S, Haddad D, Chen CH, et al. Novel therapy for anaplastic thyroid carcinoma cells using an oncolytic vaccinia virus carrying the human sodium iodide symporter. Surgery. 2011;150:1040-1047.

8. Smanik PA, Liu Q, Furminger TL, et al. Cloning of the human sodium iodide symporter. Biochem Biophys Res Commun. 1996;226:339-345.

9. Hingorani M, Spitzweg C, Vassaux G, et al. The biology of the sodium iodide symporter and its potential for targeted gene delivery. Curr Cancer Drug Targets. 2010;10:242-267.

10. Frentzen A, Yu YA, Chen N, et al. Anti-VEGF single-chain antibody GLAF-1 encoded by oncolytic vaccinia virus significantly enhances antitumor therapy. Proc Natl Acad Sci USA. 2009;106:12915-12920.
11. Serganova I, Ponomarev V, Blasberg R. Human reporter genes: potential use in clinical studies. Nucl Med Biol. 2007;34:791-807.

12. Barton KN, Stricker H, Brown SL, et al. Phase I study of noninvasive imaging of adenovirus-mediated gene expression in the human prostate. Mol Ther. 2008; 16:1761-1769.

13. Serganova I, Blasberg R. Reporter gene imaging: potential impact on therapy. Nucl Med Biol. 2005;32:763-780.

14. Zhang Q, Yu YA, Wang E, et al. Eradication of solid human breast tumors in nude mice with an intravenously injected light-emitting oncolytic vaccinia virus. Cancer Res. 2007;67:10038-10046.

15. Guide for the Care and Use of Laboratory Animals. Washington, DC: National Academy Press; 1996.

16. Herzog H, Zilken H, Niederbremer A, Friedrich W, Muller-Gartner HW. Calculation of residence times and radiation doses using the standard PC software Excel. Eur J Nucl Med. 1997;24:1514-1521.

17. Furhang EE, Larson SM, Buranapong P, Humm JL. Thyroid cancer dosimetry using clearance fitting. J Nucl Med. 1999;40:131-136.

18. Kuruppu D, Brownell AL, Zhu A, et al. Positron emission tomography of herpes simplex virus 1 oncolysis. Cancer Res. 2007;67:3295-3300.

19. Yu YA, Shabahang S, Timiryasova TM, et al. Visualization of tumors and metastases in live animals with bacteria and vaccinia virus encoding light-emitting proteins. Nat Biotechnol. 2004;22:313-320.

20. Brader P, Kelly KJ, Chen N, et al. Imaging a genetically engineered oncolytic vaccinia virus (GLV-1h99) using a human norepinephrine transporter reporter gene. Clin Cancer Res. 2009;15:3791-3801.

21. Spitzweg C, Morris JC. The sodium iodide symporter: its pathophysiological and therapeutic implications. Clin Endocrinol (Oxf). 2002;57:559-574.

22. Dai G, Levy O, Carrasco N. Cloning and characterization of the thyroid iodide transporter. Nature. 1996;379:458-460.

23. Merron A, Baril P, Martin-Duque P, et al. Assessment of the $\mathrm{Na} / \mathrm{I}$ symporter as a reporter gene to visualize oncolytic adenovirus propagation in peritoneal tumours. Eur J Nucl Med Mol Imaging. 2010;37:1377-1385.

24. Merron A, Peerlinck I, Martin-Duque P, et al. SPECT/CT imaging of oncolytic adenovirus propagation in tumours in vivo using the $\mathrm{Na} / \mathrm{I}$ symporter as a reporter gene. Gene Ther. 2007;14:1731-1738.

25. Carlson SK, Classic KL, Hadac EM, et al. Quantitative molecular imaging of viral therapy for pancreatic cancer using an engineered measles virus expressing the sodium-iodide symporter reporter gene. AJR. 2009;192:279287.

26. Msaouel P, Iankov ID, Allen C, et al. Noninvasive imaging and radiovirotherapy of prostate cancer using an oncolytic measles virus expressing the sodium iodide symporter. Mol Ther. 2009; 17:2041-2048.

27. Goel A, Carlson SK, Classic KL, et al. Radioiodide imaging and radiovirotherapy of multiple myeloma using VSV(Delta51)-NIS, an attenuated vesicular stomatitis virus encoding the sodium iodide symporter gene. Blood. 2007;110: 2342-2350.

28. Hakkarainen T, Rajecki M, Sarparanta M, et al. Targeted radiotherapy for prostate cancer with an oncolytic adenovirus coding for human sodium iodide symporter. Clin Cancer Res. 2009;15:5396-5403.

29. Hingorani M, White CL, Zaidi S, et al. Therapeutic effect of sodium iodide symporter gene therapy combined with external beam radiotherapy and targeted drugs that inhibit DNA repair. Mol Ther. 2010;18:1599-1605.

30. Peerlinck I, Merron A, Baril P, et al. Targeted radionuclide therapy using a Wnttargeted replicating adenovirus encoding the Na/I symporter. Clin Cancer Res. 2009;15:6595-6601.

31. Dingli D, Peng KW, Harvey ME, et al. Image-guided radiovirotherapy for multiple myeloma using a recombinant measles virus expressing the thyroidal sodium iodide symporter. Blood. 2004;103:1641-1646.

32. Penheiter AR, Wegman TR, Classic KL, et al. Sodium iodide symporter (NIS)-mediated radiovirotherapy for pancreatic cancer. AJR. 2010;195:341349. 